\title{
Pulse Compression Techniques for Target Detection
}

\author{
K.L.Priyanka \\ Dept. of ECM, K.L.University \\ Guntur, India
}

\author{
Sujatha Ravichandran \\ Sc-G, RCl, \\ Hyderabad
}

\author{
N.Venkatram \\ HOD ECM, \\ K.L.University, Guntur, India
}

\begin{abstract}
LPI is the property of radar that, because of its low power, wide bandwidth, frequency variability, or other design attributes makes it difficult for it to be detected by means of a passive intercept receiver. Desirable properties of LPI based on periodic autocorrelation, ambiguity function, peak and integrated side lobes. LPI modulation techniques include frequency modulation, phase modulations. In phase modulation Barker, Frank, P4simulations are studied. The best code is opted for target detection based on desirable properties, Doppler tolerance and reduction in side lobes. When the transmitted and received signals are correlated a peak is generated as the indication of target detection. These correlated peaks are added non coherently to achieve target detection.
\end{abstract}

\section{Keywords}

Pulse compression, correlation, polyphase codes, polytime codes, side lobe, weighting windows

\section{INTRODUCTION}

LPI radar is a class of radar systems that possess certain performance characteristics that make them nearly undetectable by intercept radars. LPI radars use periodically modulated CW signals resulting in large bandwidths and small resolution cells, and are ideally suited for pulse compression. The LPI radar transmits a low power intra pulse modulated waveforms so that the range of detected target can be determined with a good range resolution. This modulation may be phase or frequency modulated pseudo random modulation.

The LPI desirable properties are periodic autocorrelation function, autocorrelation function, ambiguity function, peak and integrated side lobes. The ambiguity function (AF) is analyze the response of a matched receiver that uses transmitted function to cross-correlate the return $\mathrm{CW}$ signal and perform target detection[5]. The time side lobe levels in the autocorrelation function (ACF) and periodic autocorrelation function (PACF) helps to quantify the LPI waveform in its ability to detect targets without interfering side lobes. If the ACF has high side lobes, a second nearby target might be able to hide in a side lobe and go undetected. The PSL is a useful measure when a single point target and for distributed targets ISL is considered. The PSL values for polyphase codes are as follows

Table1.Comparison of codes based on peak side lobe level

\begin{tabular}{|c|c|c|}
\hline \multirow{2}{*}{$\begin{array}{c}\text { Types of } \\
\text { codes }\end{array}$} & \multicolumn{2}{|c|}{ Peak sidelobe level in DB } \\
\cline { 2 - 3 } Barker & ACF & PACF \\
\hline Frank & -22.3 & -22 \\
\hline P1 & -28 & Perfect \\
\hline P2 & -28 & Perfect \\
\hline P3 & -25 & -22 \\
\hline
\end{tabular}

\section{\begin{tabular}{l|l|l|}
\hline P4 & -20.15 & perfect
\end{tabular}}

Here, the perfect PSL level indicates the zero side lobes. Pulse compression radar waveforms offer several advantages over uncompressed waveforms. In pulse compression technique within transmit power limitations; range, Doppler resolution and target detection capabilities are greatly improved. A pulse compression signal modulation involves switching or keying the amplitude, frequency, phase of the carrier in accordance with the information in binary digits. Frequency-modulated pulse compression techniques involve sweeping the carrier frequency of the transmit waveform in a linear or nonlinear fashion. For easy implementation phase modulated pulse compression technique is used.

Pulse compression with phase shift keying involves the following methods Barker polyphase sequences and the Frank code, $P 1, P 2, P 3$, and $P 4$ codes, and polytime codes T1, T2, T3, and T4. Polyphase compression codes are derived from step approximation of linear frequency modulation [5].

\section{PHASE SHIFT KEYING}

\subsection{Polyphase codes}

The polyphase codes may be efficiently implemented to provide large pulse compression ratios, with normalized peak side lobes. The advantages with polyphase waveforms are ability to achieve low side lobes with weighting on receiver. The polyphase codes have good nonperiodic correlation properties.

Polyphase Barker code has a flexibility of generating phase modulated waveforms. The problem with the Barker code is having limited code length and present of side lobes, which are capable of masking of nearby targets.

Frank code is related to linear frequency modulation and Barker code. Frank has $\mathrm{N}$ frequency steps and $\mathrm{N}$ samples per frequency. The no. of samples in frank code is N2. The problem with Frank is it is only applicable to the code of perfect square length. The phase of Frank code is

$$
\emptyset_{i, j}=\frac{2 \pi}{N}(i-1)(j-1)
$$

Where $i=1,2 \ldots . N$, and $j=1,2 \ldots N$

The P1 code and P2 code are also derived from a step approximation to an LFM waveform using $\mathrm{N}$ frequency steps and $\mathrm{N}$ samples per frequency. The phases of $P 1, P 2$ codes are

$$
\begin{aligned}
\emptyset_{i, j} & =-\frac{\pi}{N}[\mathbf{N}-(\mathbf{2} \mathbf{j}-\mathbf{1})][(\mathbf{j}-\mathbf{1})+(\mathbf{i}-\mathbf{1})] \\
\emptyset_{i, j} & =\left[\frac{\pi}{2} \cdot \frac{\mathbf{N}-\mathbf{1}}{\mathbf{N}}-\frac{\pi}{\mathbf{N}}(\mathbf{i}-\mathbf{1})\right](\mathbf{N}+\mathbf{1}-\mathbf{2} \mathbf{j})
\end{aligned}
$$

Where $\mathrm{i}=1,2 \ldots \mathrm{N}$, and $\mathrm{j}=1,2 \ldots \mathrm{N}$. The $P 2$ code has a property of being palindrome.

The $P 3$ code and $P 4$ code are derived by sampling an LFM waveform. The phases of $\mathrm{P} 3$ and $\mathrm{P} 4$ codes are

$$
\begin{aligned}
& \emptyset_{i}=\frac{\pi}{N_{c}}(i-1)^{2} \\
& \emptyset_{i}=\frac{\pi}{N_{c}}(i-1)^{2}-\pi(i-1)
\end{aligned}
$$




\subsection{Polytime Codes}

Polytime codes have fixed phase states with varying time period at each phase state. $T 1(n), T 2(n)$ waveforms are generated by using stepped frequency model. T3(n),T4(n) waveforms are approximations to a linear frequency modulation waveform. The time spent at any given phase is reduced by increasing the number of phase states. The phase state duration changes as a function of time. The generations of polytime waveforms are complicated.

Here, polyphase codes of frank and P4 are considered. For maximal Doppler shift $\mathrm{F}=15$, maximal delay $\mathrm{T}=1.1$, no. of positive Doppler shift $\mathrm{K}=80$, no. of delay shifts on each side $\mathrm{N}=80$, over sampling ratio $\mathrm{SR}=10$; the three characteristics of the signal: amplitude, phase and frequency; ambiguity plot, autocorrelation, periodic auto correlation plots are as follows.

\subsection{Frank code ambiguity analysis}

Frank polyphase pulse compression of length 16 is used.

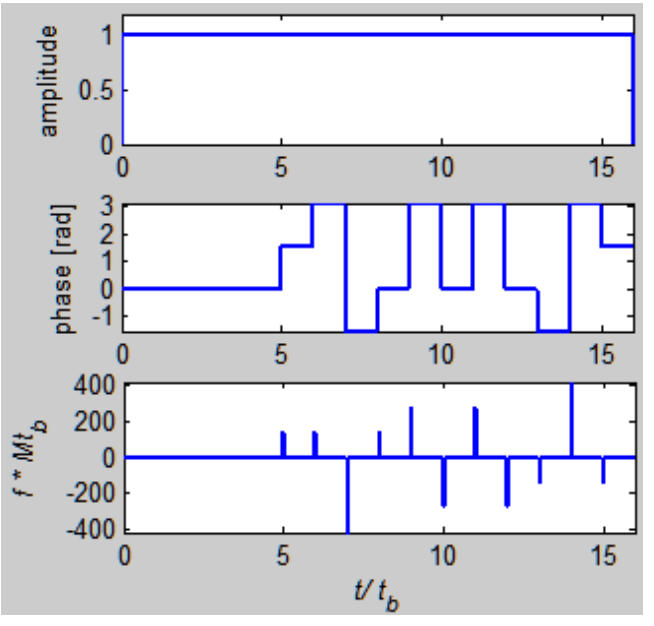

Fig 2.1.1: characteristics of the signal

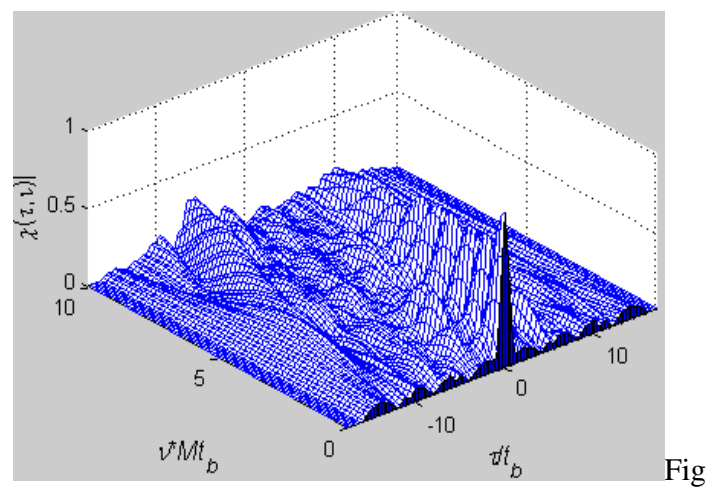

2.1.2: Ambiguity plot

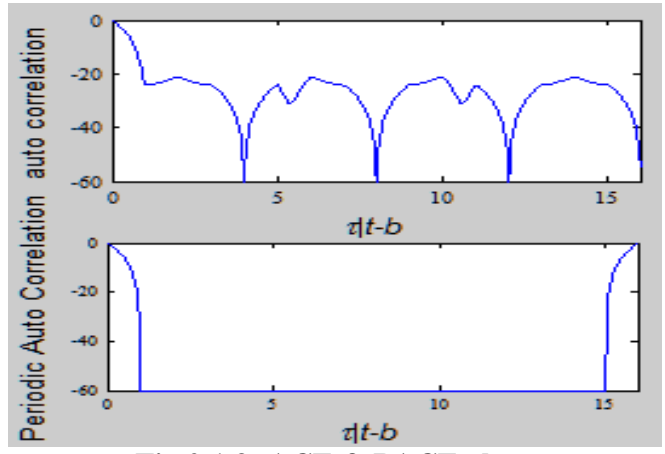

Fig 2.1.3: ACF \& PACF plots

\section{2. $\mathbf{P 4}$ code ambiguity analysis}

$P 4$ polyphase pulse compression of length 25 is used

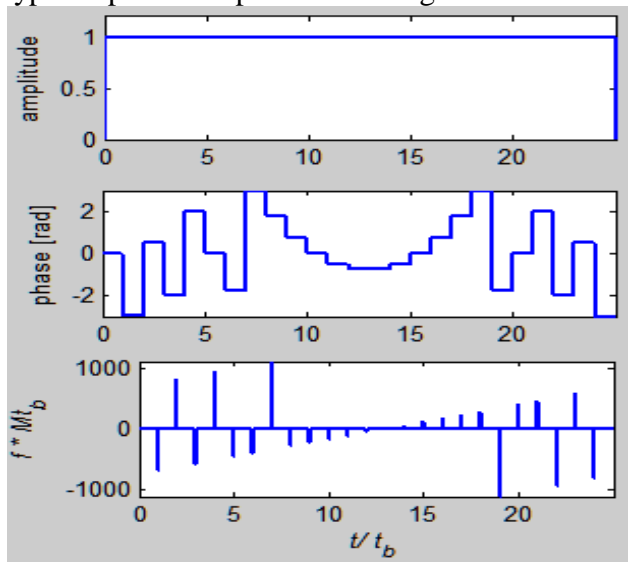

Fig 2.2.1: characteristics of the signal

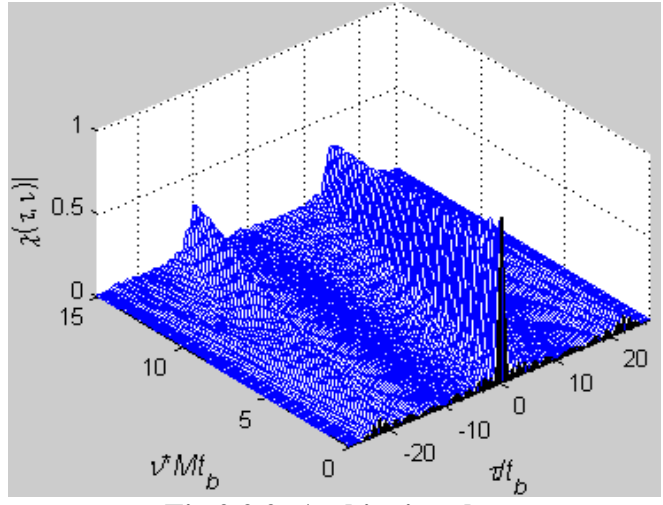

Fig 2.2.2: Ambiguity plot

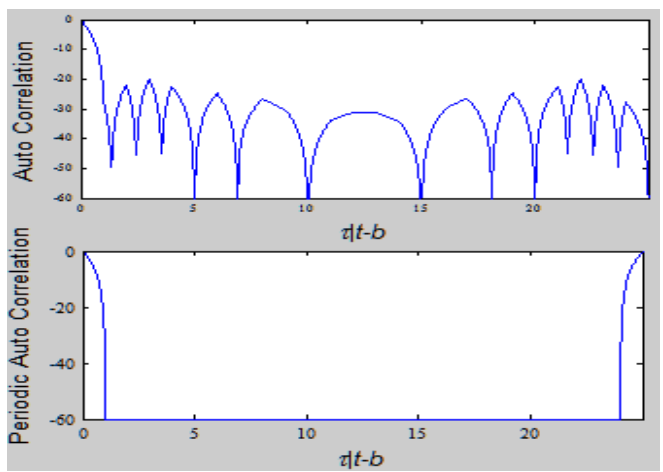

Fig 2.2.3: ACF \& PACF plots 
The side lobe levels of $P 4$ are smaller compared to the frank polyphase codes. Both frank and $p 4$ have perfect PACF values.

\section{REDUCTION OF SIDE LOBES}

The successful application of pulse compression techniques depends largely on reduction of the range side lobes associated with the compressed pulse waveform. Range side lobes mask weak targets in the presence of stronger ones. This has been accomplished either by shaping the transmitted pulse envelope or by providing a suitable band shaping network in the receiver.

To reduce the Doppler side lobes it is necessary to modify the reference signal with a weighting function $w(t)$ that converts the receiver from a matched receiver to a mismatched receiver. Amplitude weighting may be used in the receive filter to reduce side lobes, which uses the linear relation between instantaneous frequency and time along the pulse.

Interpulse weighting mitigates Doppler side lobes; Intrapulse weighting mitigates range side lobes. Hamming and Hann window functions are used to mitigate the range side lobes [3]. The weightings can be combined to reduce both the range and Doppler side lobes. However, to allow fixedamplitude transmission, the weighting can be concentrated in receiver side only.

\subsection{Frank code}

Here the Frank code of length 16 is used for weighting technique on receiver side for reduction of side lobes.

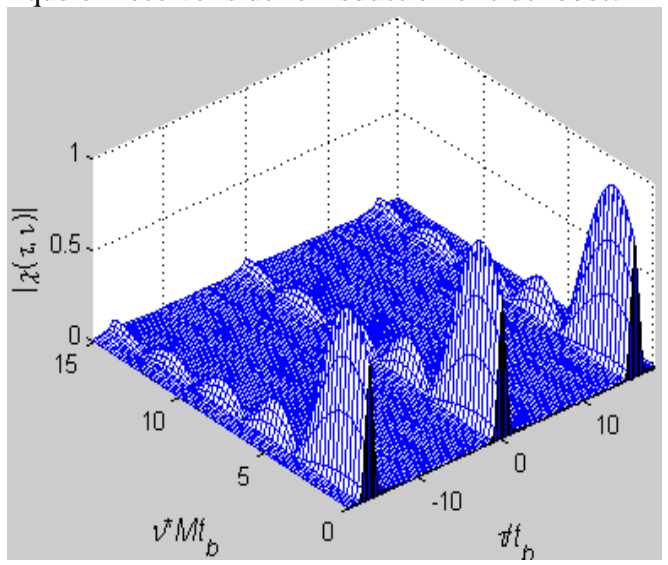

Fig 3.1.1cross ambiguity plot of rectangular window function

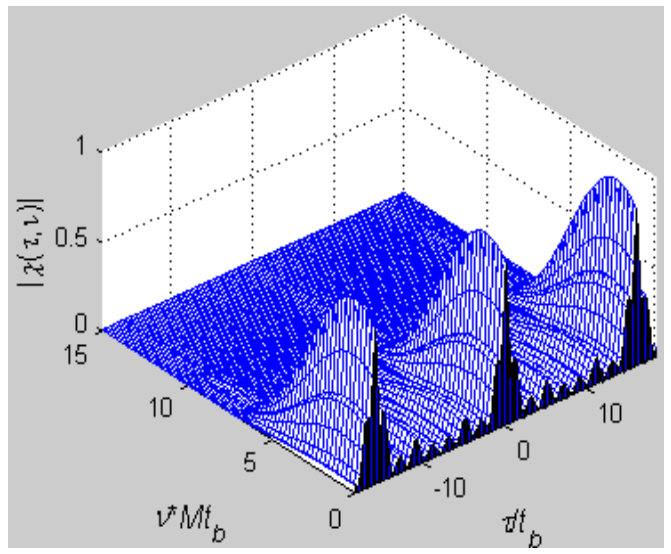

Fig 3.1.2: cross ambiguity plot of hann window function

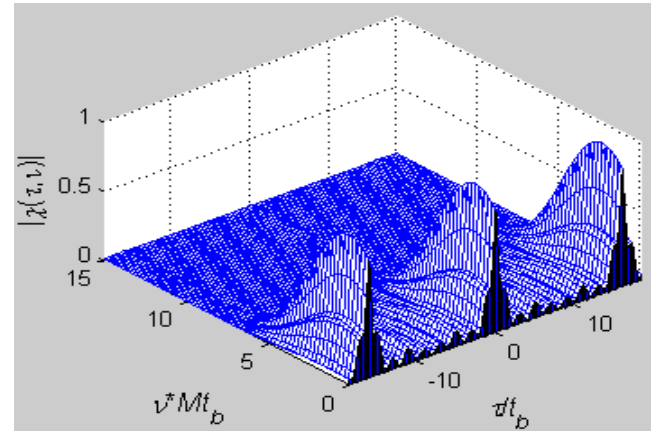

Fig3.1.3: cross ambiguity plot of hamming window function

Fig 3.1.1 shows the presence of side lobes in frank polyphase code when using rectangular window function. So, hamming and hann window functions are used in receiver side to reduce the range side lobes. In comparison between hamming and hann window functions, hamming window function successfully reduce the range side lobes. But in the Frank polyphase code the main lobes are not clear and delay side lobes are also present so $P 4$ polyphase code is used

\subsection{P4 code}

P4 polyphase pulse compression of length 25 is used for weighting technique on receiver side for reduction of side lobes.

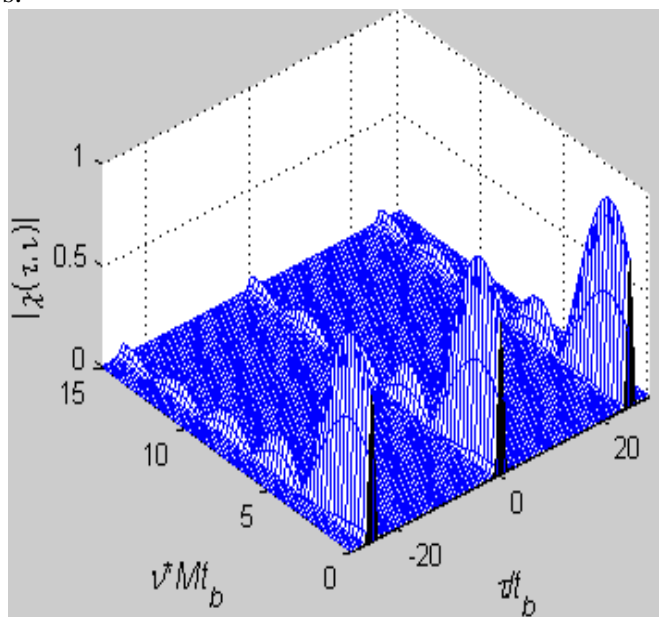

Fig 3.2.1: cross ambiguity plot of rectangular window

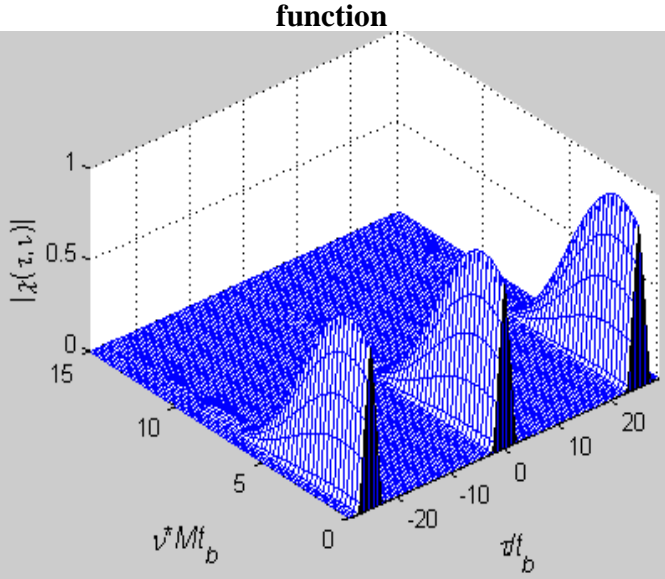

Fig 3.2.2: cross ambiguity plot of hann window function 


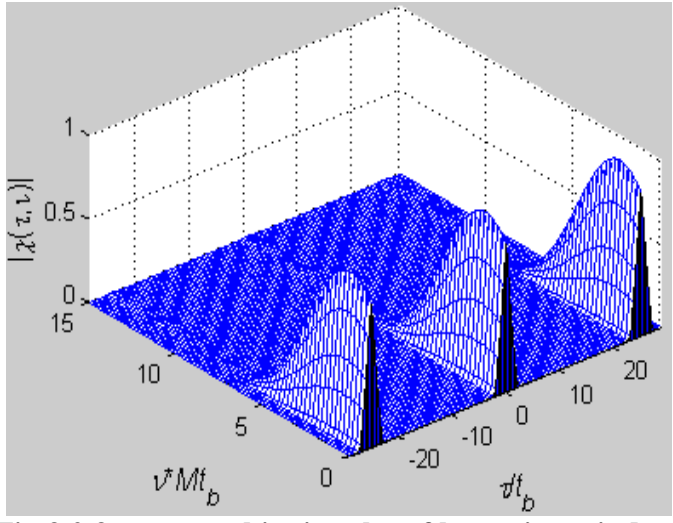

Fig 3.2.3: cross ambiguity plot of hamming window function

Fig3.2.3 shows the complete reduction of both delay and range side lobes using the hamming window function on receiver side and also widening of the main lobe. The main lobe are also clear when compare to Frank polyphase code.

\section{DOPPLER TOLERANCE}

"GOOD" codes are defined as those having one main peak in their autocorrelation function and minimum side peaks [4]. Frank, P4 codes are used for target detection. The plots are correlated outputs of signal and code

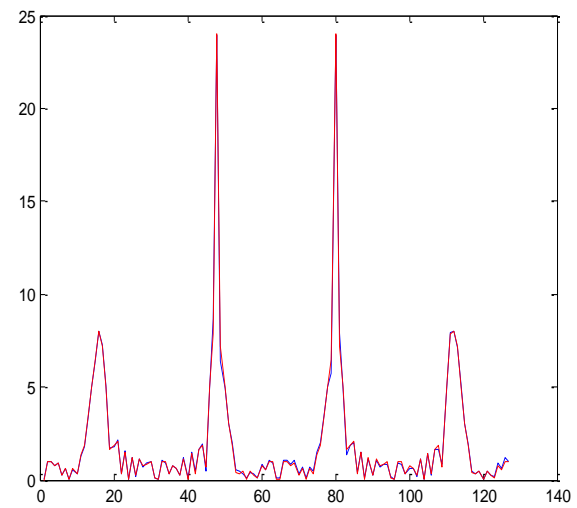

Fig 4.1: $\mathrm{P} 4$ in presence of doppler $2 \mathrm{Khz}$

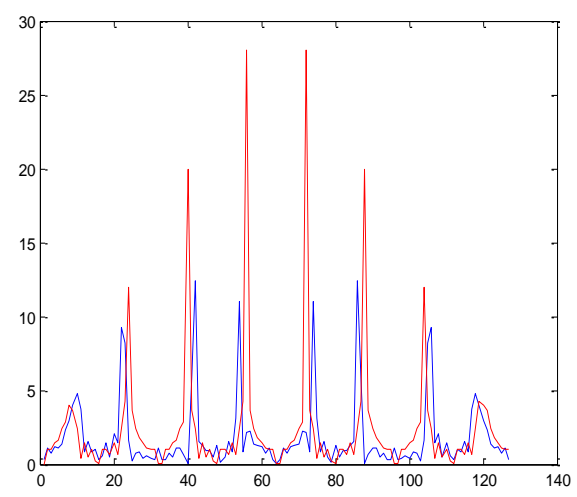

Fig 4.2: Frank in presence of doppler $2 \mathrm{Khz}$

By comparing these plots $p 4$ is best suited for target detection when Doppler is present. The peak in frank code is shifted in presence of Doppler and magnitude value is also varied.

\section{TARGET DETECTION}

To detect the target the correlation outputs are added non coherently. Hamming window technique and $P 4$ code is used for target detection. By using window technique the side lobes are reduced and width of main lobe is increases. The plots with and without windowing are as follows
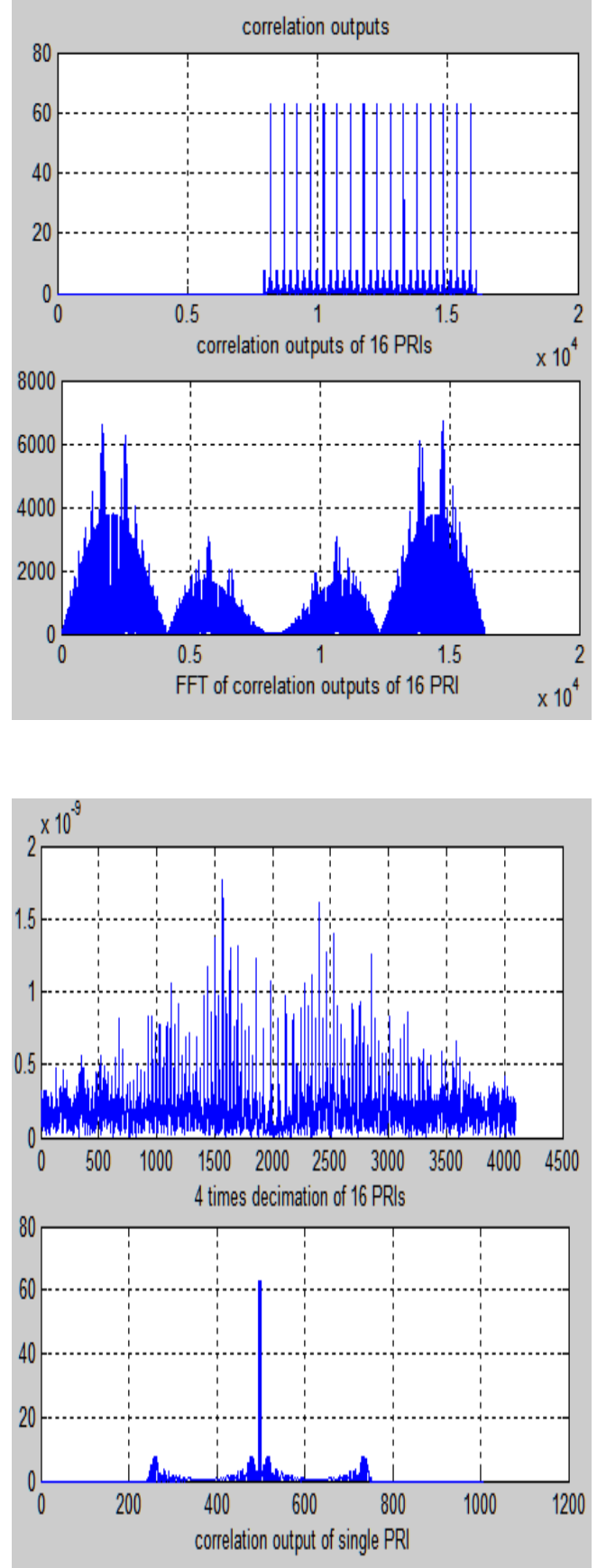

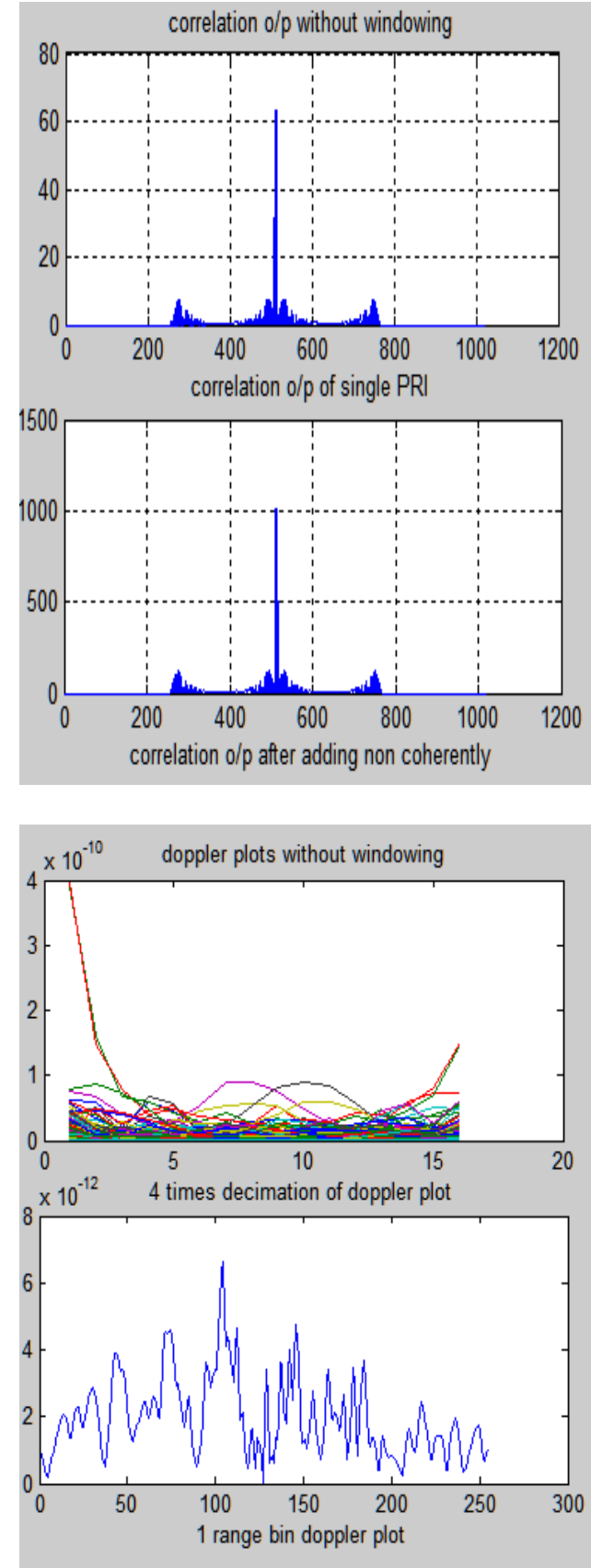

Fig 5.1 without windowing

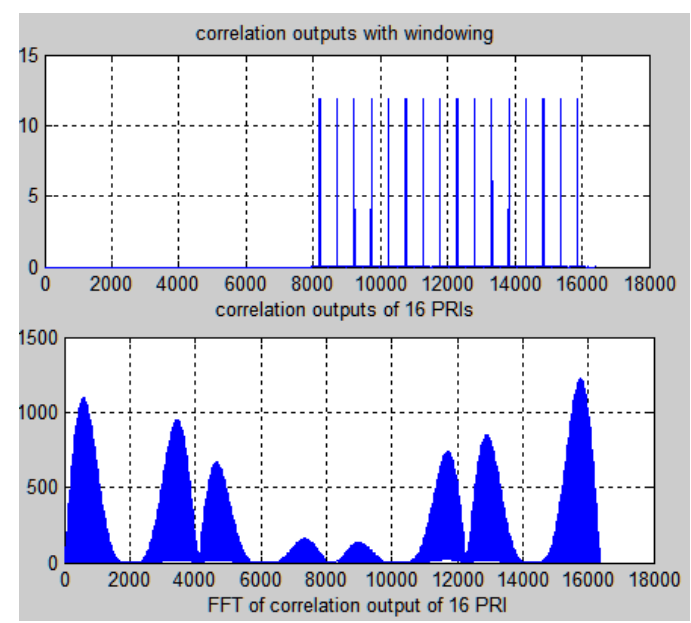

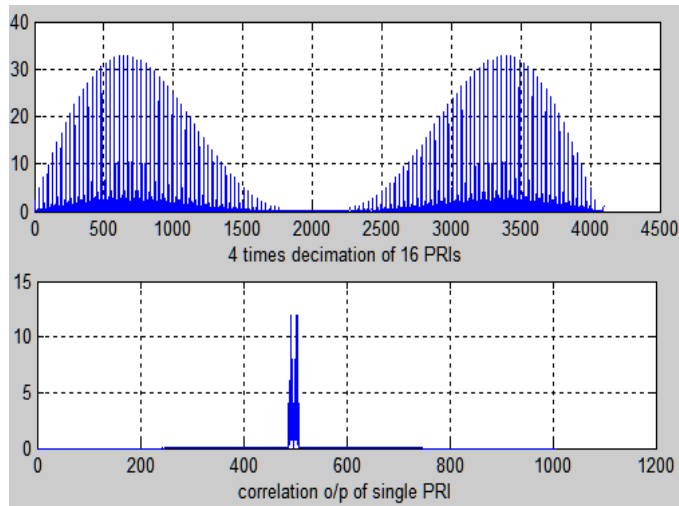
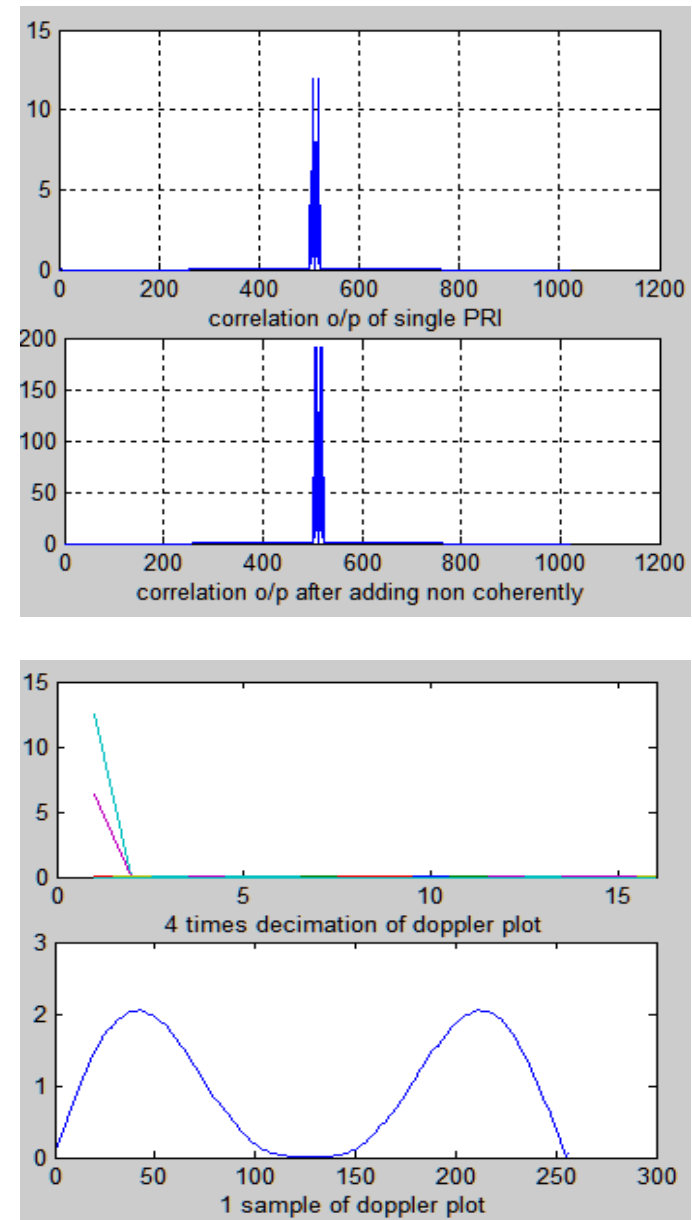

Fig 5.2 with windowing

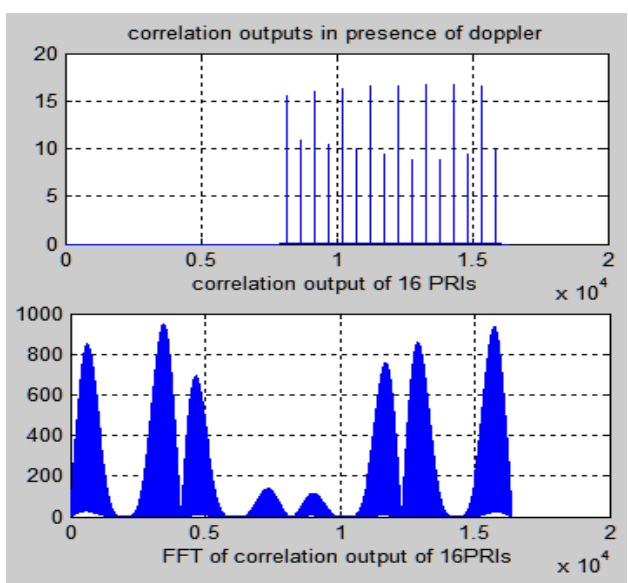



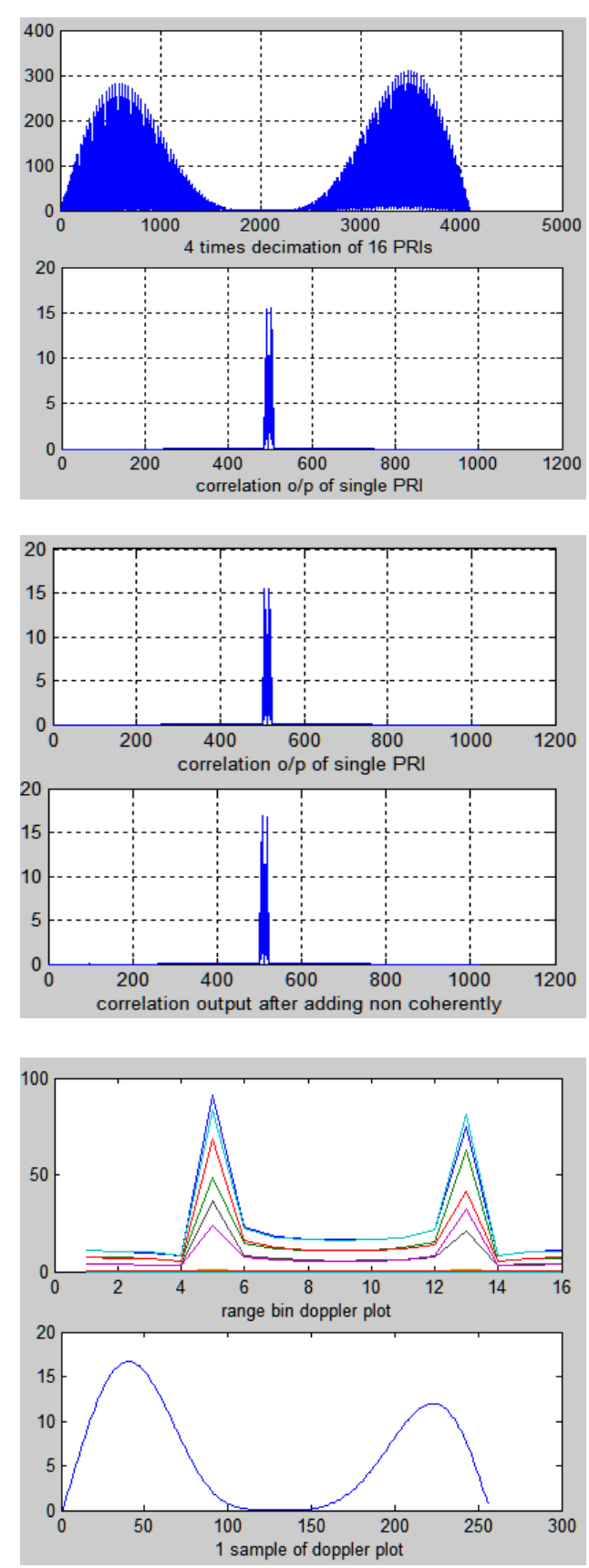

Fig 5.3 correlated outputs in presence of Doppler
From the above graphs we can conclude that in the presence of Doppler, windowing the peak value of non coherent addition value is decreases randomly.

\section{CONCLUSION}

Pulse compression is key technique for LPI radar target detection. Here the both polyphase and polytime codes are studied. In polyphase codes, Barker, Frank, P4 codes have been simulated and their characteristics studied. Barker code is eliminated because of limited code length.

Using P4 code, the target detectablity and the Doppler tolerant feature have been seen. To reduce the side lobes, Hamming window technique is used. The increase in main lobe width has been observed.

To meet the requirements of environmental changes, code agility can be used by changing the codes on a pulse to pulse basis or update to update.

\section{REFERENCES}

[1]. M. I. Skolnik, "Radar Handbook", McGraw-Hill, New York, NY, 1970.

[2]. Bassem R. Mahafza Atef Z. Elsherbeni, "Simulations for Radar System Design”Chapter 05, July 2003.

[3]. Nedav Levanon and Eli Mozeson, "Radar Signals", Wiley, 2004.

[4]. R.L. Frank, "Polyphase codes with good nonperiodic correlation properties," IEEE Transactions on Information Theory, pp. 43-45, January 1963.

[5]. P. E. Pace, "Detecting and classifying low probability of intercept radar", Artech House, 2004.

[6]. Nedav Levanon and Eli Mozeson, "Matlab code for plotting ambiguity functions", IEEE Transactions on Aero space and Electronic System, vol.38, No.3, 10641068, 2002.

[7]. Ashok S. Mudukutore, V. Chandrasekar, "Pulse Compression for Weather Radars", IEEE Transactionson Geosciences andRemote Sensing, Vol.36, No.1, January 1998.

[8]. Jarmo Lund_en, Liisa Terho, Visa Koivunen "Classifying Pulse Compression Radar Waveforms Using Time Frequency Distributions", 2005 Conference on Information Sciences and Systems, The Johns Hopkins University, March 16-18, 2005. 